\title{
Local and global bifurcation of steady states to a general Brusselator model
}

Zhongzi Zhao ${ }^{1}$ and Ruyun Ma ${ }^{1 *}$

\section{*Correspondence:}

mary@nwnu.edu.cn

1 Department of Mathematics,

Northwest Normal University,

Lanzhou, P.R. China

\section{Abstract}

In this paper, we consider the local and global bifurcation of nonnegative nonconstant solutions of a general Brusselator model

$$
\begin{cases}-d_{1} \Delta u=a-(b+1) f(u)+u^{2} v, & x \in \Omega, \\ -d_{2} \Delta v=b f(u)-u^{2} v, & x \in \Omega, \\ \frac{\partial u}{\partial n}=\frac{\partial v}{\partial n}=0, & x \in \partial \Omega,\end{cases}
$$

where $d_{1}, d_{2}, a>0$ are fixed parameters with $d_{2}>d_{1}, b>0$ is a bifurcation parameter; $f \in C([0, \infty),[0, \infty))$ is a strictly increasing function and $f^{\prime}\left(f^{-1}(a)\right) \in(0, \infty)$. Moreover, via the Rabinowitz bifurcation theorem, we obtain the global structure of nonconstant solutions under the condition that $\frac{f(s)}{s^{2}}$ is nonincreasing in $(0, \infty)$.

MSC: 34B15; 34C23; 35B45

Keywords: Brusselator model; Nonconstant steady state solutions; Local bifurcation; Global bifurcation

\section{Introduction}

In 1968, Prigogine and Lefever [15] introduced firstly the Brusselator model for a chemical reaction-diffusion of self-catalysis as follows:

$$
\begin{cases}\frac{\partial u}{\partial t}-d_{1} \Delta u=a-(b+1) u+u^{2} v, & x \in \Omega, t>0, \\ \frac{\partial v}{\partial t}-d_{2} \Delta v=b u-u^{2} v, & x \in \Omega, t>0, \\ \frac{\partial u}{\partial n}=\frac{\partial v}{\partial n}=0, & x \in \partial \Omega, t>0,\end{cases}
$$

where $\Omega \subset \mathbb{R}^{N}(N \geq 1)$ is a smooth and bounded domain, $n$ denotes the outward unit normal vector on $\partial \Omega, u$ and $v$ represent the concentration of two intermediary reactants having the diffusion rates $d_{1}, d_{2} \in(0, \infty)$ with $d_{2}>d_{1}, a, b>0$ are the fixed concentrations. Indeed, (1.1) has been extensively investigated in the last decades from both analytical and numerical point of view (see $[1-8,11-14,17,18])$. Most of the authors are interested in 
finding spatially nonconstant solutions of the equilibrium problem

$$
\begin{cases}-d_{1} \Delta u=a-(b+1) u+u^{2} v, & x \in \Omega, \\ -d_{2} \Delta v=b u-u^{2} v, & x \in \Omega, \\ \frac{\partial u}{\partial n}=\frac{\partial v}{\partial n}=0, & x \in \partial \Omega .\end{cases}
$$

In $[3,4,13,14]$, they obtained the existence or nonexistence of the nonconstant solutions of (1.2) by a priori estimate and topological degree theory. Peng and Wang [13] considered the following problem:

$$
\begin{cases}-\theta \Delta u=\lambda\left(1-(b+1) u+b u^{2} v\right), & x \in \Omega, \\ -\Delta v=\lambda a^{2}\left(u-u^{2} v\right), & x \in \Omega, \\ \partial_{n} u=\partial_{n} v=0, & x \in \partial \Omega,\end{cases}
$$

and proved the nonexistence for nonconstant of (1.3) for either small $\lambda$, large $\theta$, or small $b$. Note that $[3,4,13,14]$ only studied the existence and nonexistence of nonnegative nonconstant solutions of (1.2). They could not get the global structure of the nonconstant solutions due to the limitations of the tools used. $\mathrm{Ma}$ and $\mathrm{Hu}$ [11] applied the Rabinowitz bifurcation theorem to get the global structure of nonconstant solutions of (1.2). Inspired by [11], we will consider the new, more general form of the Brusselator model:

$$
\begin{cases}-d_{1} \Delta u=a-(b+1) f(u)+u^{2} v, & x \in \Omega, \\ -d_{2} \Delta v=b f(u)-u^{2} v, & x \in \Omega, \\ \frac{\partial u}{\partial n}=\frac{\partial v}{\partial n}=0, & x \in \partial \Omega,\end{cases}
$$

where $d_{1}, d_{2}, a>0$ are fixed parameters and $d_{2}>d_{1}, b>0$ is a bifurcation parameter. Clearly, $f(u)=\frac{f(u)}{u} \cdot u$, then (1.4) is seen to be equivalent to

$$
\begin{cases}-d_{1} \Delta u=a-(b+1) \frac{f(u)}{u} \cdot u+u^{2} v, & x \in \Omega, \\ -d_{2} \Delta v=b \frac{f(u)}{u} \cdot u-u^{2} v, & x \in \Omega, \\ \frac{\partial u}{\partial n}=\frac{\partial v}{\partial n}=0, & x \in \partial \Omega .\end{cases}
$$

Compared with problem (1.2), $\frac{f(u)}{u}$ can be regarded as a variable coefficient. It is well known that the linear terms $(b+1) u$ and $b u$ in (1.2) cannot withstand any small perturbation. In fact, (1.5) has been widely applied in chemical and biological fields. We will study the local and global behavior of nonnegative nonconstant solutions of (1.4) under the following assumptions:

(H1) $f \in C([0, \infty),[0, \infty))$ is a strictly increasing function.

(H2) $f^{\prime}\left(f^{-1}(a)\right) \in(0, \infty)$.

(H3) $\frac{f(s)}{s^{2}}$ is nonincreasing in $(0, \infty)$.

Remark 1.1 If $f(u)=u$, then (1.4) will reduce to (1.2). However, if $f(u)=u+u^{2}$, a perturbation term is added to $b u$ and $(b+1) u$ in (1.2). It is easy to see that this small perturbation leads to the results in [11] that are not available. 
The rest of the paper is organized as follows: In Sect. 2, we give a priori estimate and some preliminary results. Section 3 is devoted to studying the local bifurcation of nonnegative nonconstant solutions of (1.4) with $\mathrm{N}=1$ under conditions (H1)-(H2). Finally, in Sect. 4, we add condition (H3) to obtain the global bifurcation of nonnegative nonconstant solutions of (1.4) with $\mathrm{N}=1$.

\section{Preliminary results}

At first, let us look for the constant solution of (1.4). To get it, it suffices to look for the constant solution of the following problem:

$$
\begin{cases}a-(b+1) f(u(x))+u^{2}(x) v(x)=0, & x \in \Omega, \\ b f(u(x))-u^{2}(x) v(x)=0, & x \in \Omega .\end{cases}
$$

By (H1), problem (2.1) has a unique solution $\left(f^{-1}(a), \frac{a b}{\left[f^{-1}(a)\right]^{2}}\right)$. Obviously, this is the unique solution of (1.4).

Basic to a priori estimate of the solutions of (1.4) is the following result which is due to Lou and Ni (see [9, Proposition 2.2] or [10, Lemma 2.1]).

Lemma 2.1 Let $g \in C^{1}(\bar{\Omega} \times \mathbb{R})$.

(1) If $w \in C^{2}(\Omega) \cap C^{1}(\bar{\Omega})$ satisfies

$$
\Delta w+g(x, w) \geq 0 \quad \text { in } \Omega, \quad \frac{\partial w}{\partial n} \leq 0 \quad \text { on } \partial \Omega,
$$

and $w\left(x_{0}\right)=\max _{\bar{\Omega}} w$, then $g\left(x_{0}, w\left(x_{0}\right)\right) \geq 0$.

(2) If $w \in C^{2}(\Omega) \cap C^{1}(\bar{\Omega})$ satisfies

$$
\Delta w+g(x, w) \leq 0 \quad \text { in } \Omega, \quad \frac{\partial w}{\partial n} \geq 0 \quad \text { on } \partial \Omega,
$$

and $w\left(x_{0}\right)=\min _{\bar{\Omega}} w$, then $g\left(x_{0}, w\left(x_{0}\right)\right) \leq 0$.

Now, we will give a priori estimate of the nonnegative nonconstant solutions of (1.4).

Lemma 2.2 Let (H1), (H2), and (H3) hold. Then any nonnegative nonconstant solution $(u, v)$ of (1.4) satisfies

$$
\begin{aligned}
& f^{-1}\left(\frac{a}{b+1}\right) \leq u(x) \leq f^{-1}(a)+\frac{d_{2}}{d_{1}} \cdot \frac{a b}{(b+1)\left[f^{-1}\left(\frac{a}{b+1}\right)\right]^{2}}, \quad x \in \Omega, \\
& \frac{b f\left(f^{-1}(a)+\frac{d_{2}}{d_{1}} \cdot \frac{a b}{(b+1)\left[f^{-1}\left(\frac{a}{b+1}\right)\right]^{2}}\right)}{\left[f^{-1}(a)+\frac{d_{2}}{d_{1}} \cdot \frac{a b}{(b+1)\left[f^{-1}\left(\frac{a}{b+1}\right)\right]^{2}}\right]^{2}} \leq v(x) \leq \frac{a b}{(b+1)\left[f^{-1}\left(\frac{a}{b+1}\right)\right]^{2}}, \quad x \in \Omega .
\end{aligned}
$$

Proof Let $x_{0} \in \bar{\Omega}$ be the minimum point of $u$. From (2) of Lemma 2.1, we have

$$
\begin{aligned}
& a-(b+1) f\left(u\left(x_{0}\right)\right)+u^{2}\left(x_{0}\right) v\left(x_{0}\right) \leq 0, \\
& a-(b+1) f\left(u\left(x_{0}\right)\right) \leq 0,
\end{aligned}
$$




$$
f\left(u\left(x_{0}\right)\right) \geq \frac{a}{b+1} .
$$

Then $u\left(x_{0}\right) \geq f^{-1}\left(\frac{a}{b+1}\right)$ by (H1), and so

$$
u(x) \geq u\left(x_{0}\right) \geq f^{-1}\left(\frac{a}{b+1}\right), \quad x \in \Omega .
$$

Let $x_{1} \in \bar{\Omega}$ be the maximum point of $v$. Similarly, we can get that

$$
b f\left(u\left(x_{1}\right)\right)-u^{2}\left(x_{1}\right) v\left(x_{1}\right) \geq 0 .
$$

Then

$$
v(x) \leq v\left(x_{1}\right) \leq \frac{b f\left(u\left(x_{1}\right)\right)}{u^{2}\left(x_{1}\right)}, \quad x \in \Omega .
$$

Combining this with (2.2), from (H3), we show

$$
v(x) \leq \frac{b f\left(u\left(x_{1}\right)\right)}{u^{2}\left(x_{1}\right)} \leq \frac{a b}{(b+1)\left[f^{-1}\left(\frac{a}{b+1}\right)\right]^{2}}, \quad x \in \Omega .
$$

Let $w=d_{1} u+d_{2} v$. Then it follows from (1.4) that

$$
\begin{cases}-\triangle w(x)=a-f(u(x)), & x \in \Omega, \\ \frac{\partial w}{\partial n}=0, & x \in \partial \Omega .\end{cases}
$$

Now, let $x_{2} \in \bar{\Omega}$ be the maximum point of $w$. By (1) of Lemma 2.1, $a-f\left(u\left(x_{2}\right)\right) \geq 0$. Then, from (H1), it is easy to see $u\left(x_{2}\right) \leq f^{-1}(a)$. Combining this with (2.3), we know that, for any $x \in \bar{\Omega}$,

$$
d_{1} u(x) \leq w(x) \leq w\left(x_{2}\right) \leq d_{1} f^{-1}(a)+d_{2} \cdot \frac{a b}{(b+1)\left[f^{-1}\left(\frac{a}{b+1}\right)\right]^{2}},
$$

then

$$
u(x) \leq f^{-1}(a)+\frac{d_{2}}{d_{1}} \cdot \frac{a b}{(b+1)\left[f^{-1}\left(\frac{a}{b+1}\right)\right]^{2}}, \quad x \in \Omega .
$$

From (2) of Lemma 2.1, if $x_{3} \in \bar{\Omega}$ is the minimum point of $v$, then

$$
b f\left(u\left(x_{3}\right)\right)-u^{2}\left(x_{3}\right) v\left(x_{3}\right) \leq 0,
$$

and

$$
v(x) \geq v\left(x_{3}\right) \geq \frac{b f\left(u\left(x_{3}\right)\right)}{u^{2}\left(x_{3}\right)} \geq \frac{b f\left(f^{-1}(a)+\frac{d_{2}}{d_{1}} \cdot \frac{a b}{(b+1)\left[f^{-1}\left(\frac{a}{b+1}\right)\right]^{2}}\right)}{\left[f^{-1}(a)+\frac{d_{2}}{d_{1}} \cdot \frac{a b}{(b+1)\left[f^{-1}\left(\frac{a}{b+1}\right)\right]^{2}}\right]^{2}}, \quad x \in \Omega .
$$

Consequently, the proof is completed. 
For any fixed $l>0$. It is well known that

$$
\left\{\begin{array}{l}
-\varphi^{\prime \prime}=\mu \varphi, \quad x \in(0, l) \\
\varphi^{\prime}(0)=\varphi^{\prime}(l)=0
\end{array}\right.
$$

has a sequence of simple eigenvalues

$$
\mu_{j}=\left(\frac{j \pi}{l}\right)^{2}, \quad j=0,1,2, \ldots
$$

the corresponding eigenfunctions are

$$
\varphi_{j}(x)= \begin{cases}1, & j=0, \\ \cos \left(\frac{j \pi x}{l}\right), & j>0 .\end{cases}
$$

Let

$$
X:=\left\{(u, v): u, v \in C^{2}[0, l], u^{\prime}(0)=u^{\prime}(l)=v^{\prime}(0)=v^{\prime}(l)=0\right\}, \quad Y:=L^{2}(0, l) \times L^{2}(0, l) .
$$

$X$ constitutes the Banach space in $C^{2}$ norm and $Y$ is a Hilbert space based on the inner product

$$
\left(w_{1}, w_{2}\right)_{Y}=\left(u_{1}, u_{2}\right)_{L^{2}(0, l)}+\left(v_{1}, v_{2}\right)_{L^{2}(0, l)},
$$

where $w_{1}=\left(u_{1}, v_{1}\right), w_{2}=\left(u_{2}, v_{2}\right) \in Y$.

\section{Local bifurcation}

For simplicity, let us consider (1.4) with $N=1$ and $\Omega=(0, l)$,

$$
\begin{cases}-d_{1} u^{\prime \prime}=a-(b+1) f(u)+u^{2} v, & x \in(0, l), \\ -d_{2} v^{\prime \prime}=b f(u)-u^{2} v, & x \in(0, l), \\ u^{\prime}(0)=u^{\prime}(l)=v^{\prime}(0)=v^{\prime}(l)=0 . & \end{cases}
$$

Clearly, $\bar{w}:=\left(f^{-1}(a), \frac{a b}{\left[f^{-1}(a)\right]^{2}}\right)$ is the unique constant solution of (3.1).

Define the mapping $P:(0, \infty) \times X \rightarrow Y$,

$$
P(b, w)=\left(\begin{array}{c}
d_{1} u^{\prime \prime}+a-(b+1) f(u)+u^{2} v \\
d_{2} v^{\prime \prime}+b f(u)-u^{2} v
\end{array}\right) .
$$

For the fixed $b>0, w=(u, v)$ is a solution of (3.1) if and only if $(b, w)$ is a zero-point of $P$. Note that $P(b, \bar{w})=\left(\begin{array}{l}0 \\ 0\end{array}\right)$ for any $b>0$, since $\bar{w}$ is the constant solution of (3.1). Let

$$
\left\{\begin{array}{l}
u=f^{-1}(a)+\sum_{k=1}^{\infty} \varepsilon^{k} u_{k} \\
v=\frac{a b}{\left.f^{-1}(a)\right]^{2}}+\sum_{k=1}^{\infty} \varepsilon^{k} v_{k}
\end{array}\right.
$$


and

$$
b=b_{0}+\sum_{k=1}^{\infty} \varepsilon^{k} b_{k}
$$

We also have to Taylor expand $f$ at the point $f^{-1}(a)$. The purpose of the rest of this section is to solve $b_{0}$ and prove that $\left(b_{0}, \bar{w}\right)$ is the bifurcation point of $P(b, w)=\left(\begin{array}{l}0 \\ 0\end{array}\right)$. First of all, we substitute (3.2) and (3.3) into (3.1) and let the higher-order term of $\varepsilon$ be equal to 0 . Then we can get the problem

$$
\begin{cases}-d_{1} u_{1}^{\prime \prime}=\left(-b_{0} f^{\prime}\left(f^{-1}(a)\right)-f^{\prime}\left(f^{-1}(a)\right)+\frac{2 a b_{0}}{f^{-1}(a)}\right) u_{1}+\left[f^{-1}(a)\right]^{2} v_{1}, & x \in(0, l), \\ -d_{2} v_{1}^{\prime \prime}=\left(b_{0} f^{\prime}\left(f^{-1}(a)\right)-\frac{2 a b_{0}}{f^{-1}(a)}\right) u_{1}-\left[f^{-1}(a)\right]^{2} v_{1}, & x \in(0, l), \\ u_{1}^{\prime}(0)=u_{1}^{\prime}(l)=v_{1}^{\prime}(0)=v_{1}^{\prime}(l)=0 . & \end{cases}
$$

In (3.4), by using the undetermined coefficient method, it follows that

$$
\begin{aligned}
b_{0} & =\frac{f^{-1}(a)\left\{d_{1} d_{2} \mu_{j}^{2}+d_{2} f^{\prime}\left(f^{-1}(a)\right) \mu_{j}+d_{1}\left[f^{-1}(a)\right]^{2} \mu_{j}+\left[f^{-1}(a)\right]^{2} f^{\prime}\left(f^{-1}(a)\right)\right\}}{2 a d_{2} \mu_{j}-d_{2} f^{-1}(a) f^{\prime}\left(f^{-1}(a)\right) \mu_{j}} \\
& :=b_{0}^{j}, \quad j=1,2, \ldots
\end{aligned}
$$

Moreover, it is not difficult to prove that (3.4) has a nontrivial solution $\left(u_{1}, v_{1}\right)$,

$$
\left\{\begin{array}{l}
u_{1}=c_{1}(j) \cos \left(\frac{j \pi}{l} x\right)=c_{1}(j) \varphi_{j}(x), \quad c_{1}(j)=-\frac{d_{2} \mu_{j}}{d_{1} \mu_{j}+f^{\prime}\left(f^{-1}(a)\right)} \\
v_{1}=\cos \left(\frac{j \pi}{l} x\right)=\varphi_{j}(x) .
\end{array}\right.
$$

Next, we substitute (3.2) and (3.3) into (3.1) and let the higher-order term of $\varepsilon^{2}$ be equal to 0 , then (3.1) becomes the following system:

$$
\begin{cases}d_{1} u_{2}^{\prime \prime}+\left(-b_{0} f^{\prime}\left(f^{-1}(a)\right)-f^{\prime}\left(f^{-1}(a)\right)+\frac{2 a b_{0}}{f^{-1}(a)}\right) u_{2}+\left[f^{-1}(a)\right]^{2} v_{2}=-F_{1}, & x \in(0, l), \\ d_{2} v_{2}^{\prime \prime}+\left(b_{0} f^{\prime}\left(f^{-1}(a)\right)-\frac{2 a b_{0}}{f^{-1}(a)}\right) u_{2}-\left[f^{-1}(a)\right]^{2} v_{2}=F_{1}, & x \in(0, l), \\ u_{2}^{\prime}(0)=u_{2}^{\prime}(l)=v_{2}^{\prime}(0)=v_{2}^{\prime}(l)=0 & \end{cases}
$$

where

$$
F_{1}=\left(\frac{2 a b_{1}}{f^{-1}(a)}-b_{1} f^{\prime}\left(f^{-1}(a)\right)\right) u_{1}+2 f^{-1}(a) u_{1} v_{1}+\frac{a b_{0}}{\left[f^{-1}(a)\right]^{2}} u_{1}^{2} .
$$

In order to solve $b_{1}$ from (3.5), let us consider the following adjoint system of the homogeneous system related to (3.5):

$$
\begin{cases}d_{1} y_{2}^{\prime \prime}+\left(-b_{0} f^{\prime}\left(f^{-1}(a)\right)-f^{\prime}\left(f^{-1}(a)\right)+\frac{2 a b_{0}}{f^{-1}(a)}\right) y_{2} & \\ +\left(b_{0} f^{\prime}\left(f^{-1}(a)\right)-\frac{2 a b_{0}}{f^{-1}(a)}\right) z_{2}=0, & x \in(0, l), \\ d_{2} z_{2}^{\prime \prime}+\left[f^{-1}(a)\right]^{2} y_{2}-\left[f^{-1}(a)\right]^{2} z_{2}=0, & x \in(0, l), \\ y_{2}^{\prime}(0)=y_{2}^{\prime}(l)=z_{2}^{\prime}(0)=z_{2}^{\prime}(l)=0 . & \end{cases}
$$


It is not difficult to verify that (3.6) has a solution $\left(y_{2}, z_{2}\right)$,

$$
\left\{\begin{array}{l}
y_{2}=c_{2}(j) \cos \left(\frac{j \pi}{l} x\right)=c_{2}(j) \varphi_{j}(x), \quad c_{2}(j)=1+\frac{d_{2} \mu_{j}}{\left[f^{-1}(a)\right]^{2}} \\
z_{2}=\cos \left(\frac{j \pi}{l} x\right)=\varphi_{j}(x) .
\end{array}\right.
$$

It is obvious that the vectors $\left(-F_{1}, F_{1}\right)$ and $\left(y_{2}, z_{2}\right)$ should be orthogonal in $L^{2}(0, l)$ by virtue of the solvability condition for (3.5), i.e.,

$$
\int_{0}^{l}\left(z_{2}-y_{2}\right) F_{1} d x=0
$$

In fact,

$$
\begin{aligned}
& \int_{0}^{l}\left(z_{2}-y_{2}\right) F_{1} d x \\
& \quad=\int_{0}^{l}-\frac{d_{2} \mu_{j}}{\left[f^{-1}(a)\right]^{2}}\left[\left(\frac{2 a b_{1}}{f^{-1}(a)}-b_{1} f^{\prime}\left(f^{-1}(a)\right)\right) u_{1}\right. \\
& \left.\quad+2 f^{-1}(a) u_{1} v_{1}+\frac{a b_{0}}{\left[f^{-1}(a)\right]^{2}} u_{1}^{2}\right] \cos \left(\frac{j \pi x}{l}\right) d x \\
& \quad=0 .
\end{aligned}
$$

Let us substitute $b_{0}, u_{1}$, and $v_{1}$ into (3.7), then $b_{1}^{j}:=b_{1}=0$, and so $F_{1}$ will reduce to

$$
\begin{aligned}
F_{1} & =2 f^{-1}(a) c_{1}(j) \varphi_{j}^{2}(x)+\frac{a b_{0}}{\left[f^{-1}(a)\right]^{2}} c_{1}^{2}(j) \varphi_{j}^{2}(x) \\
& =2 f^{-1}(a) c_{1}(j) \cos ^{2}\left(\frac{j \pi}{l} x\right)+\frac{a b_{0}}{\left[f^{-1}(a)\right]^{2}} c_{1}^{2}(j) \cos ^{2}\left(\frac{j \pi}{l} x\right) \\
& =f^{-1}(a) c_{1}(j)\left(\cos \left(\frac{2 j \pi}{l} x\right)+1\right)+\frac{1}{2} \cdot \frac{a b_{0}}{\left[f^{-1}(a)\right]^{2}} c_{1}^{2}(j)\left(\cos \left(\frac{2 j \pi}{l} x\right)+1\right) \\
& =\frac{1}{2}\left[2 f^{-1}(a) c_{1}(j)+\frac{a b_{0}}{\left[f^{-1}(a)\right]^{2}} c_{1}^{2}(j)\right]+\frac{1}{2}\left[2 f^{-1}(a) c_{1}(j)+\frac{a b_{0}}{\left[f^{-1}(a)\right]^{2}} c_{1}^{2}(j)\right] \varphi_{2 j}(x) .
\end{aligned}
$$

Therefore, a particular solution $\left(u_{2}, v_{2}\right)$ of (3.5) can be obtained as follows:

$$
\left\{\begin{array}{l}
u_{2}=a_{1}(j)+a_{2}(j) \cos \left(\frac{2 j \pi}{l} x\right)=a_{1}(j)+a_{2}(j) \varphi_{2 j}(x), \\
v_{2}=a_{3}(j)+a_{4}(j) \cos \left(\frac{2 j \pi}{l} x\right)=a_{3}(j)+a_{4}(j) \varphi_{2 j}(x),
\end{array}\right.
$$

where

$$
\begin{aligned}
a_{2}(j)= & \left(\left[f^{-1}(a)\right]^{2} c_{1} d_{2} \mu_{2 j}+\frac{a b_{0} d_{2} \mu_{2 j} c_{1}^{2}}{2 f^{-1}(a)}\right) \\
& /\left(d_{1} d_{2} \mu_{2 j}^{2} f^{-1}(a)+f^{\prime}\left(f^{-1}(a)\right) f^{-1}(a)\left(\left[f^{-1}(a)\right]^{2}+d_{2} \mu_{2 j}\left(1+b_{0}\right)\right)\right. \\
& \left.-\left(2 a b_{0} d_{2}-d_{1}\left[f^{-1}(a)\right]^{3}\right) \mu_{2 j}\right),
\end{aligned}
$$




$$
\begin{aligned}
& a_{1}(j)=0, \quad a_{3}(j)=-\frac{c_{1}}{\left[f^{-1}(a)\right]^{2}}\left(f^{-1}(a)+\frac{a b_{0}}{2\left[f^{-1}(a)\right]^{2}} c_{1}\right), \\
& a_{4}(j)=-\frac{d_{1} \mu_{2 j}+f^{\prime}\left(f^{-1}(a)\right)}{d_{2} \mu_{2 j}} a_{2}(j) .
\end{aligned}
$$

Since $b_{1}=0$, we have to solve $b_{2}$. We substitute (3.2) and (3.3) into (3.1) and let the higher-order term of $\varepsilon^{3}$ be equal to 0 , then a problem similar to (3.5) is obtained:

$$
\begin{cases}d_{1} u_{3}^{\prime \prime}+\left(-b_{0} f^{\prime}\left(f^{-1}(a)\right)-f^{\prime}\left(f^{-1}(a)\right)+\frac{2 a b_{0}}{f^{-1}(a)}\right) u_{3}+\left[f^{-1}(a)\right]^{2} v_{3}=-F_{2}, & x \in(0, l), \\ d_{2} v_{3}^{\prime \prime}+\left(b_{0} f^{\prime}\left(f^{-1}(a)\right)-\frac{2 a b_{0}}{f^{-1}(a)}\right) u_{3}-\left[f^{-1}(a)\right]^{2} v_{3}=F_{2}, & x \in(0, l), \\ u_{3}^{\prime}(0)=u_{3}^{\prime}(l)=v_{3}^{\prime}(0)=v_{3}^{\prime}(l)=0, & \end{cases}
$$

where

$$
F_{2}=\left(-b_{2} f^{\prime}\left(f^{-1}(a)\right)+\frac{2 a b_{2}}{f^{-1}(a)}\right) u_{1}+2 f^{-1}(a) u_{1} v_{2}+2 f^{-1}(a) u_{2} v_{1}+u_{1}^{2} v_{1}+\frac{2 a b_{0}}{\left[f^{-1}(a)\right]^{2}} u_{1} u_{2} .
$$

Clearly, (3.6) is also the adjoint system of the homogeneous system related to (3.8), then

$$
\int_{0}^{l}\left(z_{2}-y_{2}\right) F_{2} d x=0
$$

and, according to values of $u_{1}, u_{2}, v_{1}$, and $v_{2}$, we have

$$
\begin{aligned}
& \int_{0}^{l}\left(z_{2}-y_{2}\right) F_{2} d x \\
& =\left(-b_{2} f^{\prime}\left(f^{-1}(a)\right)+\frac{2 a b_{2}}{f^{-1}(a)}\right) c_{1} \int_{0}^{l} \cos \left(\frac{j \pi}{l} x\right) d x \\
& \quad+2 f^{-1}(a) \int_{0}^{l} c_{1}\left(a_{3}+a_{4} \cos \left(\frac{2 j \pi}{l} x\right)\right) \cdot \cos ^{2}\left(\frac{j \pi}{l} x\right) d x \\
& \quad+2 f^{-1}(a) a_{2} \int_{0}^{l} \cos \left(\frac{2 j \pi}{l} x\right) \cdot \cos ^{2}\left(\frac{j \pi}{l} x\right) d x+\int_{0}^{l} c_{1}^{2} \cos ^{4}\left(\frac{j \pi}{l} x\right) d x \\
& \quad+\frac{2 a b_{0} c_{1} a_{2}}{\left[f^{-1}(a)\right]^{2}} \int_{0}^{l} \cos ^{2}\left(\frac{j \pi}{l} x\right) \cdot \cos \left(\frac{2 j \pi}{l} x\right) d x \\
& =0 .
\end{aligned}
$$

Thus,

$$
b_{2}=-\frac{\left[f^{-1}(a)\right]^{3}\left(2 c_{1} a_{3}+c_{1} a_{4}+a_{2}\right)+3 c_{1}^{2}\left[f^{-1}(a)\right]^{2}+a b_{0}^{j} c_{1} a_{2}}{2 a c_{1} f^{-1}(a)-f^{\prime}\left(f^{-1}(a)\right)\left[f^{-1}(a)\right]^{2} c_{1}}:=b_{2}^{j} \neq 0, \quad j=1,2, \ldots
$$

From the above analysis, we obtain the main result of this section.

Theorem 3.1 Assume that $(\mathrm{H} 1)$ and $(\mathrm{H} 2)$ hold. Then, for any positive integer $j,\left(b_{0}^{j}, \bar{w}\right)$ is a bifurcation point of $P(b, w)=\left(\begin{array}{l}0 \\ 0\end{array}\right)$. Moreover, there is a nontrivial solution $\phi(\varepsilon)=$ $(b(\varepsilon), u(\varepsilon), v(\varepsilon))$ of $(3.1)$ if $\varepsilon$ is small enough, where $b, u$, and $v$ are continuous with respect 
to $\varepsilon$, and

$$
\begin{aligned}
& u(\varepsilon)=f^{-1}(a)+\varepsilon c_{1}(j) \varphi_{j}+\varepsilon^{2}\left(a_{1}(j)+a_{2}(j) \varphi_{2 j}\right)+o\left(\varepsilon^{2}\right), \\
& v(\varepsilon)=\frac{a b}{\left[f^{-1}(a)\right]^{2}}+\varepsilon \varphi_{j}+\varepsilon^{2}\left(a_{3}(j)+a_{4}(j) \varphi_{2 j}\right)+o\left(\varepsilon^{2}\right), \\
& b(\varepsilon)=b_{0}^{j}+\varepsilon^{2} b_{2}^{j}+o\left(\varepsilon^{2}\right) .
\end{aligned}
$$

The set of zero-points of P constitutes two curves in a neighborhood of the bifurcation point $\left(b_{0}^{j}, \bar{w}\right)$.

Let $\mathbb{C}$ be the closure of the nonconstant solution set of $P(b, w)=\left(\begin{array}{l}0 \\ 0\end{array}\right), \Gamma_{j}$ be a connected component of $\mathbb{C} \cup\left\{\left(b_{0}^{j}, \bar{w}\right)\right\}$, and $\left(b_{0}^{j}, \bar{w}\right) \in \Gamma_{j}$. In a small neighborhood of bifurcation point $\left(b_{0}^{j}, \bar{w}\right)$, the curve $\Gamma_{j}$ is determined by the eigenfunction $\varphi_{j}$, where $\varphi_{j}$ has $j$ zeros in the interval $[0, l]$.

\section{Global bifurcation}

Theorem 4.1 Let (H1), (H2), and (H3) hold. If $\mu_{j} \neq \frac{\left[f^{-1}(a)\right]^{2}}{d_{2}}, j=1,2, \ldots$, then the projection of continuum $\Gamma_{j}$ is unbounded on the b-axis.

Proof (3.1) can be written as follows:

$$
\begin{cases}-u^{\prime \prime}(x)=g(u, v), & x \in(0, l), \\ -v^{\prime \prime}(x)=h(u, v), & x \in(0, l), \\ u^{\prime}(0)=u^{\prime}(l)=v^{\prime}(0)=v^{\prime}(l)=0, & \end{cases}
$$

where

$$
g(u, v)=\frac{1}{d_{1}}\left(a-(b+1) f(u)+u^{2} v\right), h(u, v)=\frac{1}{d_{2}}\left(b f(u)-u^{2} v\right) .
$$

Let $\tilde{u}=u-f^{-1}(a), \tilde{v}=v-\frac{a b}{\left.f^{-1}(a)\right]^{2}}$. Then (4.1) is equivalent to the following problem:

$$
\begin{cases}-\tilde{u}^{\prime \prime}=g_{0} \tilde{u}+g_{1} \tilde{v}+\tilde{g}(\tilde{u}, \tilde{v}), & x \in(0, l), \\ -\tilde{v}^{\prime \prime}=h_{0} \tilde{u}+h_{1} \tilde{v}+\tilde{h}(\tilde{u}, \tilde{v}), & x \in(0, l), \\ \tilde{u}^{\prime}(0)=\tilde{u}^{\prime}(l)=\tilde{v}^{\prime}(0)=\tilde{v}^{\prime}(l)=0, & \end{cases}
$$

where $\tilde{g}$ and $\tilde{h}$ are higher-order terms of $\tilde{u}, \tilde{v}$, and

$$
\begin{aligned}
& g_{0}=\left.g_{u}(u, v)\right|_{\left(f^{-1}(a), \frac{a b}{\left[f^{-1}(a)\right]^{2}}\right)}=\frac{1}{d_{1}}\left(-(b+1) f^{\prime}\left(f^{-1}(a)\right)+\frac{2 a b}{f^{-1}(a)}\right), \\
& g_{1}=\left.g_{v}(u, v)\right|_{\left(f^{-1}(a), \frac{a b}{\left[f^{-1}(a)\right]^{2}}\right)}=\frac{\left[f^{-1}(a)\right]^{2}}{d_{1}}, \\
& h_{0}=\left.h_{u}(u, v)\right|_{\left(f^{-1}(a), \frac{a b}{\left[f^{-1}(a)\right]^{2}}\right)}=\frac{1}{d_{2}}\left(b f^{\prime}\left(f^{-1}(a)\right)-\frac{2 a b}{f^{-1}(a)}\right), \\
& h_{1}=\left.h_{v}(u, v)\right|_{\left(f^{-1}(a), \frac{a b}{\left[f^{-1}(a)\right]^{2}}\right)}=-\frac{\left[f^{-1}(a)\right]^{2}}{d_{2}} .
\end{aligned}
$$


In this way, we convert the constant solution $\bar{w}=\left(f^{-1}(a), \frac{a b}{\left.f^{-1}(a)\right]^{2}}\right)$ of (3.1) to the trivial solution $\theta=(0,0)$ of $(4.2)$.

Let $H_{1}: Y \rightarrow X$ and $H_{2}: Y \rightarrow X$ be the inverse of operators $\frac{f^{\prime}\left(f^{-1}(a)\right)}{d_{1}} I-\frac{d^{2}}{d x^{2}}$ and $\frac{\left[f^{-1}(a)\right]^{2}}{d_{2}} I-$ $\frac{d^{2}}{d x^{2}}$ with Neumann boundary conditions, respectively. Set $U=(\tilde{u}, \tilde{v})$,

$$
\begin{aligned}
K(b) U= & \left(\frac{1}{d_{1}}\left[-b f^{\prime}\left(f^{-1}(a)\right)+\frac{2 a b}{f^{-1}(a)}\right] H_{1}(\tilde{u})\right. \\
& \left.+\frac{\left[f^{-1}(a)\right]^{2}}{d_{1}} H_{1}(\tilde{v}), \frac{1}{d_{2}}\left[b f^{\prime}\left(f^{-1}(a)\right)-\frac{2 a b}{f^{-1}(a)}\right] H_{2}(\tilde{u})\right), \\
W(U)= & \left(H_{1}(\tilde{g}(\tilde{u}, \tilde{v})), H_{2}(\tilde{h}(\tilde{u}, \tilde{v}))\right) .
\end{aligned}
$$

It can be verified that (4.2) is equivalent to

$$
U=K(b) U+W(U)
$$

in $X$. For any fixed number $b>0, K(b)$ and $W(U)$ are linear compact operators in $X$ and $W(U)=o(\|U\|)$. By the Rabinowitz global bifurcation theorem [16], we need to verify

(i) 1 is an eigenvalue of $K\left(b_{0}^{j}\right)$ and its algebraic multiplicity is 1 ;

(ii) the index of $I-K(b)-W$ changes when $b$ crosses $b_{0}^{j}$.

Now, we will prove (i). Suppose $\Psi=\left(\begin{array}{c}\xi \\ \psi\end{array}\right), \xi=\sum a_{j} \varphi_{j}, \psi=\sum c_{j} \varphi_{j}$. Let

$$
(K(b)-I) \Psi=\left(\begin{array}{l}
0 \\
0
\end{array}\right)
$$

i.e.,

$$
\left(\begin{array}{cc}
\frac{1}{d_{1}}\left[-(b+1) f^{\prime}\left(f^{-1}(a)\right)+\frac{2 a b}{f^{-1}(a)}\right]+\frac{d^{2}}{d x^{2}} & \frac{\left.f^{-1}(a)\right]^{2}}{d_{1}} \\
\frac{1}{d_{2}}\left[b f^{\prime}\left(f^{-1}(a)\right)-\frac{2 a b}{f^{-1}(a)}\right] & -\frac{\left.f^{-1}(a)\right]^{2}}{d_{2}}+\frac{d^{2}}{d x^{2}}
\end{array}\right) \Psi=\left(\begin{array}{l}
0 \\
0
\end{array}\right),
$$

thus,

$$
\sum_{j=0}^{\infty} L_{j}\left(\begin{array}{l}
a_{j} \\
c_{j}
\end{array}\right) \varphi_{j}=\left(\begin{array}{l}
0 \\
0
\end{array}\right)
$$

where

$$
L_{j}=\left(\begin{array}{cc}
\frac{1}{d_{1}}\left[-(b+1) f^{\prime}\left(f^{-1}(a)\right)+\frac{2 a b}{f^{-1}(a)}\right]-\mu_{j} & \frac{\left[f^{-1}(a)\right]^{2}}{d_{1}} \\
\frac{1}{d_{2}}\left[b f^{\prime}\left(f^{-1}(a)\right)-\frac{2 a b}{f^{-1}(a)}\right] & -\frac{\left.f^{-1}(a)\right]^{2}}{d_{2}}-\mu_{j}
\end{array}\right) .
$$

By computation, $\operatorname{det} L_{j}=0$ if and only if $b=b_{0}^{j}$, taking $b=b_{0}^{j}$ leads to

$$
\operatorname{det} L_{j}=\operatorname{det}\left(\begin{array}{cc}
\frac{\left[f^{-1}(a)\right]^{2}}{d_{1} d_{2} \mu_{j}}\left(f^{\prime}\left(f^{-1}(a)\right)+d_{1} \mu_{j}\right) & \frac{\left[f^{-1}(a)\right]^{2}}{d_{1} d_{2} \mu_{j}} \cdot d_{2} \mu_{j} \\
-\frac{1}{d_{2}} f^{\prime}\left(f^{-1}(a)\right)-\frac{d_{1}}{d_{2}} \mu_{j} & -\mu_{j}
\end{array}\right)=0
$$


and

$$
L_{j}\left(\begin{array}{c}
a_{j} \\
c_{j}
\end{array}\right)=\left(\begin{array}{cc}
0 & 0 \\
f^{\prime}\left(f^{-1}(a)\right)+d_{1} \mu_{j} & d_{2} \mu_{j}
\end{array}\right)\left(\begin{array}{c}
a_{j} \\
c_{j}
\end{array}\right) .
$$

Then $\operatorname{ker}\left(K\left(b_{0}^{j}\right)-I\right)=\operatorname{span}(\Psi), \Psi=\left(_{f^{\prime}\left(f^{-1}(a)\right)+d_{1} \mu_{j}}^{-d_{2} \mu_{j}} \varphi_{j}\right)$. This implies that 1 is the eigenvalue of $K=K\left(b_{0}^{j}\right)$ and $\operatorname{dim} \operatorname{ker}(K-I)=1$. The algebraic multiplicity of the eigenvalue 1 is the dimension of the generalized null space $\bigcup_{i=1}^{\infty} \operatorname{ker}(K-I)^{i}$, therefore, $\operatorname{ker}(K-I) \cap \operatorname{Im}(K-I)=$ $\left\{\theta^{T}\right\}$.

Let $K^{T}$ be the transposed matrix of $K$,

$$
K^{T}=\left(\begin{array}{cc}
\frac{1}{d_{1}}\left[-b f^{\prime}\left(f^{-1}(a)\right)+\frac{2 a b}{f^{-1}(a)}\right] H_{1} & \frac{1}{d_{2}}\left[b f^{\prime}\left(f^{-1}(a)\right)-\frac{2 a b}{f^{-1}(a)}\right] H_{2} \\
\frac{\left[f^{-1}(a)\right]^{2}}{d_{1}} H_{1} & 0
\end{array}\right),
$$

and $\Psi^{*}=\left(\begin{array}{c}\xi^{*} \\ \psi^{*}\end{array}\right), \xi^{*}=\sum a_{j}^{*} \varphi_{j}, \psi^{*}=\sum c_{j}^{*} \varphi_{j}$. Suppose $\Psi^{*} \in \operatorname{ker}\left(K^{T}-I\right)$. Then

$$
\left\{\begin{array}{l}
\frac{1}{d_{1}}\left[-b f^{\prime}\left(f^{-1}(a)\right)+\frac{2 a b}{f^{-1}(a)}\right] H_{1}\left(\xi^{*}\right)+\frac{1}{d_{2}}\left[b f^{\prime}\left(f^{-1}(a)\right)-\frac{2 a b}{f^{-1}(a)}\right] H_{2}\left(\psi^{*}\right)=\xi^{*} \\
\frac{\left[f^{-1}(a)\right]^{2}}{d_{1}} H_{1}\left(\xi^{*}\right)=\psi^{*}
\end{array}\right.
$$

From the definition of $H_{1}, H_{2}$, (4.4) can also be written as

$$
\left\{\begin{aligned}
&-d_{1} d_{2} \xi^{* \prime \prime}=\left(d_{2}\left[-b f^{\prime}\left(f^{-1}(a)\right)+\frac{2 a b}{f^{-1}(a)}\right]-\left[f^{-1}(a)\right]^{2} d_{1}\right) \xi^{*} \\
&-\frac{d_{2} f^{\prime}\left(f^{-1}(a)\right)}{\left[f^{-1}(a)\right]^{2}}\left[-b f^{\prime}\left(f^{-1}(a)\right)+\frac{2 a b}{f^{-1}(a)}\right] \psi^{*}, \\
&-d_{1} \psi^{* \prime \prime}=\left[f^{-1}(a)^{2}\right] \xi^{*}-f^{\prime}\left(f^{-1}(a)\right) \psi^{*}
\end{aligned}\right.
$$

That is to say,

$$
\sum_{j=0}^{\infty} B_{j}\left(\begin{array}{c}
a_{j}^{*} \\
c_{j}^{*}
\end{array}\right) \varphi_{j}=\left(\begin{array}{l}
0 \\
0
\end{array}\right)
$$

where

$$
B_{j}=\left(\begin{array}{cc}
d_{2}\left[-b f^{\prime}\left(f^{-1}(a)\right)+\frac{2 a b}{f^{-1}(a)}\right]-\left[f^{-1}(a)\right]^{2} d_{1}-d_{1} d_{2} \mu_{j} & -\frac{d_{2} f^{\prime}\left(f^{-1}(a)\right)}{\left[f^{-1}(a)\right]^{2}}\left[-b f^{\prime}\left(f^{-1}(a)\right)+\frac{2 a b}{f^{-1}(a)}\right] \\
{\left[f^{-1}(a)\right]^{2}} & -d_{1} \mu_{j}-f^{\prime}\left(f^{-1}(a)\right)
\end{array}\right) .
$$

Similarly, det $B_{j}=0$ if and only if $b=b_{0}^{j}$, taking $b=b_{0}^{j}$ leads to

$$
\operatorname{det} B_{j}=\operatorname{det}\left(\begin{array}{cc}
\frac{\left[f^{-1}(a)\right]^{2}+d_{2} \mu_{j}}{\left[f^{-1}(a)\right]^{2} \mu_{j}}\left[f^{-1}(a)\right]^{2} & \frac{\left[f^{-1}(a)\right]^{2}+d_{2} \mu_{j}}{\left[f^{-1}(a)\right]^{2} \mu_{j}}\left(-d_{1} \mu_{j}-f^{\prime}\left(f^{-1}(a)\right)\right) \\
{\left[f^{-1}(a)\right]^{2}} & -d_{1} \mu_{j}-f^{\prime}\left(f^{-1}(a)\right)
\end{array}\right)=0
$$

and

$$
B_{j}\left(\begin{array}{c}
a_{j}^{*} \\
c_{j}^{*}
\end{array}\right)=\left(\begin{array}{cc}
0 & 0 \\
\left.f^{-1}(a)\right]^{2} & -d_{1} \mu_{j}-f^{\prime}\left(f^{-1}(a)\right)
\end{array}\right)\left(\begin{array}{c}
a_{j}^{*} \\
c_{j}^{*}
\end{array}\right) .
$$


Then $\operatorname{ker}\left(K^{*}-I\right)=\operatorname{span}\left(\begin{array}{c}d_{1} \mu_{j}+f^{\prime}\left(f^{-1}(a)\right) \\ {\left[f^{-1}(a)\right]^{2}}\end{array}\right) \varphi_{j}$. According to $\mu_{j} \neq \frac{\left[f^{-1}(a)\right]^{2}}{d_{2}}$, we obtain

$$
\begin{aligned}
\left(\Psi, \Psi^{*}\right)_{Y}= & \left(-d_{2} \mu_{j} \varphi_{j},\left(d_{1} \mu_{j}+f^{\prime}\left(f^{-1}(a)\right) \varphi_{j}\right)\right)_{L^{2}[0, l]} \\
& +\left(\left(f^{\prime}\left(f^{-1}(a)+d_{1} \mu_{j}\right)\right) \varphi_{j},\left[f^{-1}(a)\right]^{2} \varphi_{j}\right)_{L^{2}[0, l]} \\
= & \left(d_{1} \mu_{j}+f^{\prime}\left(f^{-1}(a)\right)\right)\left(\left[f^{-1}(a)\right]^{2}-d_{2} \mu_{j}\right) \int_{0}^{l} \cos ^{2}\left(\frac{j \pi}{l} x\right) d x \\
= & \frac{l}{2}\left(d_{1} \mu_{j}+f^{\prime}\left(f^{-1}(a)\right)\right)\left(\left[f^{-1}(a)\right]^{2}-d_{2} \mu_{j}\right) \neq 0 .
\end{aligned}
$$

This suggests that $\Psi \notin\left(\operatorname{ker}\left(K^{*}-I\right)\right)^{\perp}=\operatorname{Im}(K-I)$, and so (i) is proved. Now, we will prove (ii). From (i), for any $b>0, b \neq b_{0}^{j}$ and $b$ belongs to a small neighborhood of $b_{0}^{j}, K(b)-I$ : $X \rightarrow X$ is a bijection. Fix $b>0$, then $\theta$ is a solution of (4.3) and $\theta$ is isolated. From the Leray-Schauder fixed point theory, we can get

$$
\text { index }(I-K(b)-W,(b, \theta))=\operatorname{deg}(I-K(b), B, \theta)=(-1)^{\gamma},
$$

where $B$ is a sufficiently small ball centered at $\theta, \gamma$ is the sum of the algebraic multiplicity of the eigenvalues of $K(b)$ and $\gamma>1$. We are going to verify that, for $\varepsilon>0$ is small enough,

$$
\operatorname{index}\left(I-K\left(b_{0}^{j}-\varepsilon\right)-W,\left(b_{0}^{j}-\varepsilon, \theta\right)\right) \neq \operatorname{index}\left(I-K\left(b_{0}^{j}+\varepsilon\right)-W,\left(b_{0}^{j}+\varepsilon, \theta\right)\right) .
$$

If $\tau$ is an eigenvalue of $K(b)$ and $\Psi=\left(\begin{array}{l}\xi \\ \psi\end{array}\right)$ is the corresponding eigenfunction, then

$$
(K(b)-I) \Psi=\left(\begin{array}{l}
0 \\
0
\end{array}\right)
$$

i.e.,

$$
\left\{\begin{array}{l}
-\tau d_{1} \xi^{\prime \prime}=\left(-b f^{\prime}\left(f^{-1}(a)\right)+\frac{2 a b}{f^{-1}(a)}-\tau f^{\prime}\left(f^{-1}(a)\right)\right) \xi+\left[f^{-1}(a)\right]^{2} \psi \\
-\tau d_{2} \psi^{\prime \prime}=\left(b f^{\prime}\left(f^{-1}(a)\right)+\frac{2 a b}{f^{-1}(a)}\right) \xi-\left[f^{-1}(a)\right]^{2} \tau \psi
\end{array}\right.
$$

By virtue of $\xi=\sum a_{j} \varphi_{j}$ and $\psi=\sum c_{j} \varphi_{j}$, we can get

$$
\sum_{j=0}^{\infty}\left(\begin{array}{cc}
\tau \mu_{j} d_{1}+b f^{\prime}\left(f^{-1}(a)\right)-\frac{2 a b}{f^{-1}(a)}+\tau f^{\prime}\left(f^{-1}(a)\right) & -\left[f^{-1}(a)\right]^{2} \\
-b f^{\prime}\left(f^{-1}(a)\right)+\frac{2 a b}{f^{-1}(a)} & \tau \mu_{j} d_{2}+\tau\left[f^{-1}(a)\right]^{2}
\end{array}\right)\left(\begin{array}{l}
a_{j} \\
c_{j}
\end{array}\right) \varphi_{j}=\left(\begin{array}{l}
0 \\
0
\end{array}\right) .
$$

Then the characteristic equation is

$$
\begin{aligned}
& \left(d_{1} d_{2} \mu_{j}^{2}+d_{2} \mu_{j} f^{\prime}\left(f^{-1}(a)\right)+d_{1} \mu_{j}\left[f^{-1}(a)\right]^{2}+f^{\prime}\left(f^{-1}(a)\right)\left[f^{-1}(a)\right]^{2}\right) \tau^{2} \\
& \quad+b\left(d_{2} f^{\prime}\left(f^{-1}(a)\right) \mu_{j}-\frac{2 a d_{2} \mu_{j}}{f^{-1}(a)}+f^{\prime}\left(f^{-1}(a)\right)\left[f^{-1}(a)\right]^{2}-2 a f^{-1}(a)\right) \tau \\
& +\left[f^{-1}(a)\right]^{2}\left(-b f^{\prime}\left(f^{-1}(a)\right)+\frac{2 a b}{f^{-1}(a)}\right)=0, \quad j=0,1,2, \ldots
\end{aligned}
$$


If $\tau=1, b$ can be solved from (4.6):

$$
b=\frac{f^{-1}(a)\left\{d_{1} d_{2} \mu_{j}^{2}+d_{2} f^{\prime}\left(f^{-1}(a)\right) \mu_{j}+d_{1}\left[f^{-1}(a)\right]^{2} \mu_{j}+\left[f^{-1}(a)\right]^{2} f^{\prime}\left(f^{-1}(a)\right)\right\}}{2 a d_{2} \mu_{j}-d_{2} f^{-1}(a) f^{\prime}\left(f^{-1}(a)\right) \mu_{j}}=b_{0}^{j} .
$$

Therefore, by calculating the corresponding eigenvalues of (4.6), we can obtain that when $b$ passes through $b_{0}^{j}$, the number of eigenvalues of $K(b)$, which is greater than 1 , is the same and their algebraic multiplicities are equal. By plugging (4.7) into (4.6), we have

$$
\begin{aligned}
& \frac{2 a d_{2} \mu_{j}-d_{2} f^{-1}(a) f^{\prime}\left(f^{-1}(a)\right) \mu_{j}}{f^{-1}(a)} \tau^{2}+\left(f^{\prime}\left(f^{-1}(a)\right)\left(d_{2} \mu_{j}+\left[f^{-1}(a)\right]^{2}\right)\right. \\
& \left.-\frac{2 a d_{2} \mu_{j}}{f^{-1}(a)}-2 a f^{-1}(a)\right) \tau+\left[f^{-1}(a)\right]^{2}\left(-f^{\prime}\left(f^{-1}(a)\right)+\frac{2 a}{f^{-1}(a)}\right)=0 .
\end{aligned}
$$

Then

$$
\begin{aligned}
\Delta:= & \left(d_{2} f^{\prime}\left(f^{-1}(a)\right) \mu_{j}-\frac{2 a d_{2} \mu_{j}}{f^{-1}(a)}+f^{\prime}\left(f^{-1}(a)\right)\left[f^{-1}(a)\right]^{2}-2 a f^{-1}(a)\right)^{2} \\
& -4\left(2 a d_{2} \mu_{j}-d_{2} f^{-1}(a) f^{\prime}\left(f^{-1}(a)\right) \mu_{j}\right) \cdot\left(-f^{\prime}\left(f^{-1}(a)\right) f^{-1}(a)+2 a\right) \\
= & {\left[\left(d_{2} f^{\prime}\left(f^{-1}(a)\right) \mu_{j}-\frac{2 a d_{2} \mu_{j}}{f^{-1}(a)}\right)-\left(f^{\prime}\left(f^{-1}(a)\right)\left[f^{-1}(a)\right]^{2}-2 a f^{-1}(a)\right)\right]^{2}>0, }
\end{aligned}
$$

and so (4.8) has two different roots $\tau_{1}=1, \tau_{2}=\frac{\left[f^{-1}(a)\right]^{2}}{d_{2} \mu_{j}}$. Thus two things will happen:

(a) if $\mu_{j}>\frac{\left[f^{-1}(a)\right]^{2}}{d_{2}}$, then $\tau_{1}\left(b_{0}^{j}\right)=1, \tau_{2}\left(b_{0}^{j}\right)<1$;

(b) if $\mu_{j}<\frac{\left[f^{-1}(a)\right]^{2}}{d_{2}}$, then $\tau_{1}\left(b_{0}^{j}\right)=1, \tau_{2}\left(b_{0}^{j}\right)>1$.

When scenario (a) occurs, $b$ passes through $b_{0}^{j}$ and $\tau_{2}(b)<1$. From (4.6), $\tau_{1}\left(b_{0}^{j}+\varepsilon\right)>1$, $\tau_{1}\left(b_{0}^{j}-\varepsilon\right)<1$. Therefore, the matrix $K\left(b_{0}^{j}+\varepsilon\right)$ has exactly one more eigenvalue, that is, $>1$, than $K\left(b_{0}^{j}-\varepsilon\right)$ does, and its algebraic multiplicity is 1 . Then (4.5) holds. That is to say, the index jumps as $b$ goes through $b_{0}^{j}$.

When scenario (b) occurs, $b$ passes through $b_{0}^{j}$ and $\tau_{2}(b)>1$. From (4.6), $\tau_{1}\left(b_{0}^{j}+\varepsilon\right)>1$, $\tau_{1}\left(b_{0}^{j}-\varepsilon\right)<1$. Similarly, the index jumps as $b$ goes through $b_{0}^{j}$. Therefore, (ii) is true regardless of (a) or (b). Thus, by the index jump principle and [16, Theorem 1.3], it follows that there exists a connected component $\hat{\Gamma}_{j}$ of nontrivial solutions of (4.3) and $\hat{\Gamma}_{j}$ comes from the bifurcation point $\left(b_{0}^{j}, \theta\right)$. We know that $\hat{\Gamma}_{j}$ is also the connected component $\Gamma_{j}$ of the nonconstant solution of (3.1) from $\left(b_{0}^{j}, \bar{w}\right), \hat{\Gamma}_{j}$ and $\Gamma_{j}$ are both in $\mathbb{R} \times X$. By the Rabinowitz global bifurcation theorem, the connected component $\Gamma_{j}$ joins $\left(b_{0}^{j}, \bar{w}\right)$ to either $\infty$ or $\left(b_{0}^{k}, \bar{w}\right)$ in $\mathbb{R} \times X$, where $k \neq j$. We first prove that the latter situation will not happen. According to Theorem 3.1, the solution on the connected component sent from $\left(b_{0}^{j}, \bar{w}\right)$ is related to $\varphi_{j}$, and $\varphi_{j}$ has $j$ zeros in the interval $[0, l]$. In the same way, the solution on the connected component sent from $\left(b_{0}^{k}, \bar{w}\right)$ is related to $\varphi_{k}$, and $\varphi_{k}$ has $k$ zeros in the interval $[0, l]$. If the connected component sent $\Gamma_{j}$ joining $\left(b_{0}^{j}, \bar{w}\right)$ to $\left(b_{0}^{k}, \bar{w}\right)$, the solution $(b, w) \in \Gamma_{j}$ is related to both $\varphi_{j}$ and $\varphi_{k}$, which is impossible. On the other hand, Lemma 2.2 shows that, if $b=b_{c} \in(0, \infty)$, then the solutions $u$ and $v$ of (3.1) are both bounded. So the connected component $\Gamma_{j}$ will not join $\left(b_{0}^{j}, \bar{w}\right)$ to $\left(b_{c}, \infty\right)$. Therefore, the connected component $\Gamma_{j}$ can only join $\left(b_{0}^{j}, \bar{w}\right)$ to either $(\infty, \infty)$ or $(\infty, m)$, where $m \in(0, \infty)$. But in any case, the projection of continuum $\Gamma_{j}$ is unbounded on the $b$-axis. 
Acknowledgements

We are very grateful to the anonymous referees for their valuable suggestions.

Funding

This work was supported by the National Natural Science Foundation of China (No. 11671322).

\section{Abbreviations}

Not applicable.

Availability of data and materials

Data sharing not applicable to this article as no datasets were generated.

\section{Competing interests}

All of the authors of this article claim that together they have no competing interests.

\section{Authors' contributions}

The authors claim that the research was realized in collaboration with the same responsibility. All authors read and approved the last version of the manuscript.

\section{Publisher's Note}

Springer Nature remains neutral with regard to jurisdictional claims in published maps and institutional affiliations.

Received: 24 July 2019 Accepted: 19 November 2019 Published online: 29 November 2019

\section{References}

1. Brown, K.J., Davidson, F.A.: Global bifurcation in the Brusselator system. Nonlinear Anal. 24, 1713-1725 (1995)

2. Gao, C., Lv, L., Wang, Y.: Spectra of a discrete Sturm-Liouville problem with eigenparameter-dependent boundary conditions in Pontryagin space. Quaest. Math. 1, 1-26 (2019)

3. Ghergu, M.: Non-constant steady-state solutions for Brusselator type systems. Nonlinearity 21, 2331-2345 (2008)

4. Ghergu, M., Rădulescu, V:: Turing patterns in general reaction-diffusion systems of Brusselator type. Commun. Contemp. Math. 12, 661-679 (2010)

5. Guo, G., Wu, J., Ren, X.: Hopf bifurcation in general Brusselator system with diffusion. Appl. Math. Mech. 32, 1177-1186 (2011)

6. Li, B., Wang, M.: Diffusion-driven instability and Hopf bifurcation in Brusselator system. Appl. Math. Mech. 29, 825-832 (2008)

7. Li, Y: Hopf bifurcations in general systems of Brusselator type. Nonlinear Anal., Real World Appl. 28, 32-47 (2016)

8. Liao, M., Wang, Q.: Stability and bifurcation analysis in a diffusive Brusselator-type system. Int. J. Bifurc. Chaos Appl. Sci. Eng. 26, 1-11 (2016)

9. Lou, Y., Ni, W.: Diffusion, self-diffusion and cross-diffusion. J. Differ. Equ. 131, 79-131 (1996)

10. Lou, Y., Ni, W.: Diffusion vs. cross-diffusion: an elliptic approach. J. Differ. Equ. 154, 157-190 (1999)

11. Ma, M., Hu, J.: Bifurcation and stability analysis of steady states to a Brusselator model. Appl. Math. Comput. 236, 580-592 (2014)

12. Ma, S.: The stochastic Hopf bifurcation analysis in Brusselator system with random parameter. Appl. Math. Comput. 219, 306-319 (2012)

13. Peng, R., Wang, M.: Pattern formation in the Brusselator system. J. Math. Anal. Appl. 309, 151-166 (2005)

14. Peng, R., Yang, M.: On steady-state solutions of the Brusselator-type system. Nonlinear Anal. 71, 1389-1394 (2009)

15. Prigogine, I., Lefever, R.: Symmetry breaking instabilities in dissipative systems II. J. Chem. Phys. 48, 1665-1700 (1968)

16. Rabinowitz, P.H.: Some global results for nonlinear eigenvalue problems. J. Funct. Anal. 7, 487-513 (1971)

17. Tzou, J.C., Ward, M.J.: The stability and slow dynamics of spot patterns in the 2D Brusselator modal: the effect of open systems and heterogeneities. Physica D 373, 13-37 (2018)

18. Zhou, J., Mu, C.: Pattern formation of a coupled two-cell Brusselator model. J. Math. Anal. Appl. 366, 679-693 (2010)

\section{Submit your manuscript to a SpringerOpen ${ }^{\circ}$ journal and benefit from:}

- Convenient online submission

- Rigorous peer review

- Open access: articles freely available online

- High visibility within the field

- Retaining the copyright to your article

Submit your next manuscript at $\gg$ springeropen.com 